\title{
Enquête
}

Archives de la revue Enquête

3 | 1996

Interpréter, Surinterpréter

\section{La violence faite aux données}

De quelques figures de la surinterprétation en anthropologie

Abuse of data. Concerning some forms of overinterpretation in anthropology

Jean-Pierre Olivier de Sardan

\section{CpenEdition}

Journals

Édition électronique

URL : http://journals.openedition.org/enquete/363

DOI : 10.4000/enquete.363

ISSN : 1953-809X

Éditeur :

Cercom, Éditions Parenthèses

Édition imprimée

Date de publication : 1 novembre 1996

Pagination : 31-59

Référence électronique

Jean-Pierre Olivier de Sardan, «La violence faite aux données », Enquête [En ligne], 3 | 1996, mis en ligne le 11 juillet 2013, consulté le 01 mai 2019. URL : http://journals.openedition.org/enquete/363 DOI : 10.4000/enquete.363

Ce document a été généré automatiquement le 1 mai 2019. 


\title{
La violence faite aux données
}

\author{
De quelques figures de la surinterprétation en anthropologie \\ Abuse of data. Concerning some forms of overinterpretation in anthropology
}

Jean-Pierre Olivier de Sardan

1 De prime abord, le terme de surinterprétation, en sciences sociales du moins, évoque irrésistiblement un excès de sens imposé au « réel » dont on prétend rendre compte. Par mépris, par incompétence ou par négligence, le chercheur maltraite les données. Soupçonné de les ignorer ou de les travestir, il franchit les limites acceptables de ce qu'on peut imputer à la réalité décrite, et propose de celle-ci une image par trop non conforme, et, pour tout dire, «fausse ». Il sollicite à l'excès les éléments empiriques disponibles, ou produit des assertions qui n'en tiennent pas compte, voire les contredisent.

2 Cette notion spontanée de « surinterprétation » mêle de façon indissociable un jugement de valeur (la surinterprétation, c'est mal) et un jugement de fait (la surinterprétation, cela se réfute données à l'appui). Nous sommes là au cœur d'une épistémologie latente à la fois pratique et normative, qui est au fond celle que nous pratiquons tous, plus ou moins comme M. Jourdain, tant à propos des écrits des autres qu'en référence à nos propres travaux. On peut, certes, soupçonner ou dénoncer le moralisme derrière le jugement de valeur, et le positivisme derrière le jugement de fait, cela ne change rien à l'affaire : nous ne pouvons nous passer de ce type de jugement dans la pratique ordinaire de notre activité professionnelle.

3 Manifestement, cette notion de surinterprétation relève du « sens commun savant ». Mais peut-elle aussi servir de support à des réflexions méthodologiques ou épistémologiques plus argumentées, qui soient de quelque utilité dans l'exercice quotidien de nos disciplines, autrement dit qui relèveraient de cette "grounded epistemology", de cette épistémologie enracinée dans les problèmes du terrain, que nous appelons de nos vœux ? Il me semble que oui, et d'abord parce qu'elle a l'énorme avantage d'introduire à un débat fondamental dans nos disciplines que nous tentons tous d'esquiver plus ou moins, tant il est incommode. On admettra volontiers, d'un côté, qu'il n'y a aucune ligne jaune nettement tracée qui sépare l'interprétation acceptable de la surinterprétation inacceptable, et qu'aucune police épistémologique ne pourra jamais dresser de 
contravention. Mais on doit aussi admettre, d'un autre côté, que chacun d'entre nous a déjà été confronté à des "excès interprétatifs" qui l'ont fait sortir de ses gonds et auxquels il a opposé l'existence d'une « réalité » mieux connue par lui et honteusement malmenée ou ignorée ${ }^{1}$. Tout le problème est qu'il y a là deux registres de discours complètement différents et qui, en l'état normal de nos disciplines, ne sont jamais, ou presque, amenés à se confronter. Quand on affirme l'indiscernabilité ultime de l'interprétation et de la surinterprétation, on se situe pour l'essentiel dans un registre épistémologique abstrait. Quand on oppose les «faits" à leur méconnaissance scandaleuse ou à leur déformation éhontée, on se situe pour l'essentiel dans un registre critique contextuel.

Pourquoi cette sorte de schizophrénie entre un discours général qui apparaîtra pour les uns comme trop relativiste (avec un parfum d'« épistémologiquement correct»), et un discours particulier qui apparaitra pour les autres comme bassement positiviste? L'entrée par la «question de la surinterprétation » oblige à se confronter au problème fondamental du rapport entre « risque interprétatif » et « légitimation empirique » dans les sciences sociales en général et en anthropologie en particulier. Notre thèse est simple : bien qu'une délimitation incontestable de la frontière interprétation/surinterprétation soit impossible, bien que les contours de la surinterprétation ne puissent être cernés avec précision et de façon formelle, il est cependant possible de repérer - et de stigmatiser quelques foyers ${ }^{2}$ éminents de surinterprétation, qui sont autant de formes de "violence " faite aux supports empiriques dont toute recherche en science sociale s'autorise pour proposer des interprétations.

\section{Surinterprétation, sous-interprétation, mésinterprétation}

5 Surinterprétation appelle évidemment la mobilisation de termes à la fois contrastés et parents. Interprétation, surinterprétation, sous-interprétation et mésinterprétation semblent ainsi former un système sémiologique composé d'affinités, de distinctions et de nuances qu'il convient d'éclaircir. En première lecture la surinterprétation dépasserait les limites admissibles de l'interprétation. Elle s'opposerait au défaut symétrique et inverse de sousinterprétation. Elle se démarquerait enfin de la mésinterprétation, faute bénigne ou péché véniel.

En fait, cette typologie qui va du plus au moins n'est pas aussi satisfaisante qu'il y paraît. Le véritable enjeu épistémologique est avant tout le partage entre interprétation et surinterprétation, du moins si on s'en tient à une définition opératoire et non essentialiste de la surinterprétation, et si on lève les hypothèques de la mésinterprétation et de la sous-interprétation.

7 Nous considérerons donc par convention comme relevant de la surinterprétation tous les cas où apparaît une contradiction significative entre les références empiriques et les propositions interprétatives. L'essentiel ici n'est pas de savoir si «surinterprétation » est bien le mot juste ou s'il n'a pas d'autres sens (il en a), mais d'englober sous un concept unique l'ensemble des situations, pas si rares dans nos disciplines, où se conjuguent projection excessive de préconceptions et paresse méthodologique, ce qui engendre une violence faite aux données ou un déni de celles-ci. La particularité des énoncés anthropologiques surinterprétatifs (selon l'acception ici proposée du terme) est qu'il est possible de les 
récuser ou de les dé-plausibiliser non pas simplement sur des bases logiques, théoriques, ou interprétatives-alternatives, mais avant tout sur des bases empiriques, par la démonstration que des données ont été maltraitées ou non prises en compte.

Cette acception-là de "surinterprétation » n'aurait pas le même sens dans les champs de la critique littéraire, de la réflexion psychanalytique ou de l'herméneutique philosophique, qui ne travaillent que dans les empilements et les confrontations d'interprétations, sans être soumis aux contraintes d'exploration empirique méthodique qui sont celles des sciences sociales. Pour ces trois champs, la notion de surinterprétation ne peut avoir de pertinence que dotée d'une autre acception ${ }^{3}$. Si on admet notre définition de la surinterprétation en ce qu'elle a de spécifique aux sciences sociales, que deviennent alors les notions voisines de sous- et de mésinterprétation?

Soit la sous-interprétation. On pourrait certes y voir le double inversé de la surinterprétation. En ce sens il y aurait, entre le piège surinterprétatif et le piège sousinterprétatif, le niveau pertinent de l'interprétation, espace du «ni trop, ni trop peu ». Mobilisons la métaphore de l'acteur. Quand celui-ci joue " juste », c'est qu'il n'en fait pas trop (sinon on lui reprocherait de "sur-jouer »), mais qu'il en fait quand même assez (sinon on lui reprocherait de « sous-jouer »). Double refus de l'emphase et de la grisaille... Interpréter « juste »... La métaphore fait sens. Toutefois cette vision linéaire allant d'un excès à l'autre, du manque vers le trop-plein, de la sous-interprétation à la surinterprétation, avec en son milieu une interprétation légitimée par sa position centriste, est à divers égards trompeuse.

On peut en fait distinguer trois formes de la sous-interprétation. La sous-interprétation comme posture heuristique n'est pas tenable, et il suffit de renvoyer aux innombrables démonstrations qui ont fait admettre à la très grande majorité des chercheurs en sciences sociales aujourd'hui qu'il n'est pas de résultat, de recherche, de production de données, voire de fait sans interprétation. Aussi la sous-interprétation comme refus du risque interprétatif est-elle un non-sens. Certes, bien que discréditée, elle n'a pas complètement abandonné la place. Mais on peut aisément prouver qu'il ne s'agit en fait que d'interprétation honteuse et masquée, et par là même peu productive. La seconde forme de sous-interprétation, comme moment méthodologique, peut en revanche être une étape "normale » dans un processus de recherche, et, parfois même, dans une restitution de recherche. Il est des moments où l'anthropologue s'efforce de « rester au plus près des données ", d'avoir une "stratégie du profil bas » ou une "politique du document brut " (aussi délibéré ou construit soit cet effacement). Ce sont là des phases nécessaires, fécondes. Autrement dit, dans un processus de recherche clairement interprétatif de part en part, des étapes relativement sous-interprétatives sont méthodologiquement nécessaires... Transitoires, artificielles, contrôlées, elles n'ont rien à voir avec une posture sous-interprétative de principe, intenable quant au fond et improductive quant aux résultats. Enfin, il est une troisième forme de sous-interprétation, qui n'est autre que... la surinterprétation : on y reviendra.

11 Soit maintenant la mésinterprétation. Ma position serait de dire qu'il n'y a pas d'espace de la mésinterprétation. Soit on est dans l'espace des interprétations concurrentes empiriquement légitimes, c'est-à-dire dotées d'un minimum de véridicité (nous reviendrons sur cette question), et alors chacun appellera mésinterprétations les interprétations qu'il récuse (c'est-à-dire qu'il considère comme erronées, moins plausibles que les siennes). Soit on est dans l'espace des interprétations empiriquement illégitimes, c'est-à-dire quasi réfutables en véridicité (cet espace même dont nous allons décrire quelques foyers), et 
alors la mésinterprétation n'est qu'une figure de la surinterprétation, simplement conçue comme plus « soft » en ce qu'elle brutaliserait moins ce qu'on sait de la réalité sociale que telles ou telles formes « hard »...

Si l'on peut démontrer qu'une mésinterprétation anthropologique malmène les données accessibles, ne les prend pas en compte ou les mutile, elle relèvera bel et bien pour nous du domaine de la surinterprétation telle que nous l'avons définie. Si en revanche le terme de mésinterprétation est utilisé pour stigmatiser une interprétation à peu près compatible avec les données mais que l'on estime non fondée, il ne s'agit alors de rien d'autre que l'interprétation des autres, en tant qu'on ne la partage pas.

Le lecteur sceptique ne manquera pas d'objecter que tout le problème est de savoir comment départager ces deux espaces, dont il ne suffit pas de décréter l'existence. La lutte des interprétations, dans ce que j'ai appelé l'espace des interprétations empiriquement légitimes, ne prend-elle pas pour une part la forme d'objections d'ordre empirique, et chacun ne cherche-t-il pas pour une part à déstabiliser la plausibilité empirique de celui avec lequel il est en désaccord, et donc à l'exclure au moins partiellement de cet espace des interprétations empiriquement légitimes?

Un léger détour est ici nécessaire.

\section{Interprétativité et empiricité}

15 Nous partirons de deux postulats, qui ne semblent plus à démontrer à force d'avoir été réaffirmés et réargumentés par les uns et les autres: a) les sciences sociales sont fondamentalement interprétatives (corrélat: le positivisme scientiste et le naturalisme ne sont pas tenables); b) les sciences sociales sont des sciences empiriques (corrélat: l'anarchisme épistémologique et le postmodernisme ne sont pas tenables).

16 Ceci définit évidemment la ligne de crête sur laquelle évoluent l'essentiel des recherches menées dans nos disciplines. On peut le dire autrement : nous sommes dans un espace de la plausibilité (et non de la falsifiabilité), où les processus interprétatifs, aussi omniprésents soient-ils, se reconnaissent des contraintes empiriques et se donnent des procédures de (relative) vigilance méthodologique (et pas seulement de vigilance logique) qui tentent de préserver tant bien que mal une certaine adéquation entre référents empiriques et assertions interprétatives. Cette adéquation recherchée ou invoquée, sinon toujours réalisée, peut être représentée sous la forme d'un double lien qu'il s'agit d'assurer :

17 a) Lien entre le "réel de référence » et les données produites à son sujet par les opérations de recherche.

18 b) Lien entre ces données et les énoncés interprétatifs proposés. Le premier lien tente de garantir que les données produites indiquent quelque chose du « réel» et ne sont pas sans le «représenter " d'une certaine façon, aussi imparfaites soient-elles : ce lien est à dominante méthodologique. Le second lien gage autant que possible les assertions du chercheur (dans les divers registres interprétatifs possibles: descriptif, explicatif, comparatif, etc.) sur une mise en scène ou une mise en récit de ces données (sous formes d'attestations, d'exemples, de tableaux, de schémas, de cartes, de cas, etc.) : ce lien est à dominante argumentative. Tous deux sont indissociables. Dans les deux cas, il s'agit bien de légitimer des énoncés interprétatifs au nom d'un certain indice de véridicité ${ }^{4}$. L'imagination interprétative accepte en l'occurrence de se soumettre aux contraintes ou 
aux vigilances particulières que lui impose l'empiricité des sciences sociales, ce en quoi elle se distingue des " exercices libres » (à cet égard) de l'herméneutique littéraire ou de la philosophie spéculative. C'est l'ensemble « réel de référence » + « données produites à son propos »+ « usage argumentatif de ces données » qui définit le substrat empirique des sciences sociales 5 .

L'espace épistémologique propre aux sciences sociales est donc à la fois intégralement interprétatif et empiriquement contraint. On peut voir une contradiction entre ces deux postulats : il s'agit plutôt d'une difficulté. Si les sciences sociales sont interprétatives, faut-il en conclure que toutes les interprétations se valent et que seules les départagent leur virtuosité et leurs capacités de séduction intellectuelle? Non, car dans la mesure où ces interprétations sont soumises à des exigences de plausibilité empirique et de véridicité (même relatives), toutes les interprétations ne se valent pas, et chacune doit rendre des comptes. La souveraineté interprétative proclamée par le premier postulat abdique certaines de ses prérogatives au nom du second. Le rapport entre interprétation et empirie se complique d'autant plus qu'il y a interprétation et interprétation, comme il y a empirie et empirie. On ne peut sans quelque abus parler de l'« interprétation » en général (comme du «sens » en général), le terme "interprétation" recouvrant des opérations de statut cognitif très divers et pouvant s'appliquer à des référents (et des types de données) extrêmement variés ${ }^{6}$. Les modalités des interprétations ne dépendent pas seulement de la posture adoptée par le chercheur, ni de l'échelle choisie ${ }^{7}$, mais aussi des objets sociaux sur lesquels elles portent, du type de données ("émiques» et/ou "étiques", spécifiques et/ou comparatives, «qualitatives » et/ou « quantitatives ») que la stratégie de recherche adoptée permet de produire sur ces objets, des «dispositifs ${ }^{8}$ " variables où sont enchâssées les interprétations « indigènes », etc.

21 Notons simplement que, au sein de cette immense diversité des situations interprétatives, on peut, en simplifiant à l'extrême, distinguer deux pôles quant aux rapports interprétativité/empiricité. Certains énoncés sont «quasi réfutables » empiriquement ${ }^{9}$. On peut plus ou moins « prouver » que $\mathrm{X}$ a fait un contresens dans sa traduction du terme valaque « $z$ drycrhol », ou que $\mathrm{Y}$ a bien assisté au rituel de divination du 30 février et en a décrit fidèlement la topographie. D'autres énoncés sont à peu près non réfutables empiriquement. Personne ne "prouvera» jamais que $\mathrm{W}$ a eu tort en affirmant que l'histoire de l'humanité est l'histoire de l'échange, ou que $\mathrm{Z}$ a eu raison de comparer un culte de possession à la Commedia dell'Arte.

En fait, dans les cas de quasi-réfutabilité maximum, les énoncés sont descriptifs ou constatifs (c'est là un certain mode interprétatif, mais en quelque sorte a minima), et leurs référents empiriques ne sont vérifiables que dans la mesure où ils sont très circonscrits et d'ordre factuel. Nous sommes dans l'ethnographie la plus méticuleuse (ou sociographie, ou historiographie), associée à la production des données de terrain. Dans les cas de nonréfutabilité maximum, les énoncés sont hypothétiques, comparatifs ou explicatifs (il s'agit donc d'autres modes interprétatifs, plus proches de ce qu'on entend en général par «interprétation») et les référents empiriques relèvent d'espaces-temps étendus et hétérogènes: il s'agit toujours d'agrégations complexes. Nous sommes dans l'anthropologie (ou la sociologie, ou l'histoire). Ces deux extrêmes, qui sont extrêmes aussi bien au niveau des modes interprétatifs que des référents empiriques, forment les pôles d'un continuum le long duquel circule la plausibilité. Du côté de la quasi-réfutabilité maximale, l'exigence en véridicité croît, et la prise de risque interprétatif décroît. Du côté 
de la non-réfutabilité maximale, c'est l'inverse. Les assertions que nous produisons se répartissent entre ces deux pôles. Mais dans tous les cas, elles incorporent à la fois de la prise de risque interprétatif et de la légitimation empirique. Il a souvent été démontré que tout énoncé à statut empirique explicite, qui prétend rendre compte d'un état précis du monde dans un espace-temps donné, c'est-à-dire tout énoncé qui se situe dans un registre descriptif ou constatif est en fait interprétatif, bien qu'il ne se donne pas comme tel. Inversement, et ceci est moins souvent souligné, tout énoncé ouvertement interprétatif (en sciences sociales, bien sûr), et donc de type hypothétique, comparatif ou explicatif, sous-entend des légitimations d'ordre empirique, et affirme, plus ou moins explicitement, qu'il tient tels ou tels états du monde pour «vrais». Empiricité et interprétativité sont ainsi toujours mêlées, mais selon des dosages qui varient considérablement.

Ainsi, les énoncés interprétatifs, toutes échelles confondues, reposent-ils d'une façon ou d'une autre sur des présomptions ou des présupposés d'empiricité, même si c'est à des degrés divers. Les propos des chercheurs se prévalent nécessairement d'un «effet de réalité ", se targuent en permanence d'une connaissance du social par l'enquête et invoquent sans cesse des données qui sont censées témoigner de ce réel. C'est dans cette "visée " empirique, dans cette "légitimation" empirique, comme on voudra, que s'ancrent et se plaident nos interprétations, quelles que soient les précautions épistémologiques dont nous nous entourons par ailleurs.

À partir de ce contexte global résumé au pas de course, la notion de surinterprétation implique que quelque chose n'aille pas dans le couple empirie/interprétation, que l'on en fasse dire trop aux données, qu'on ne tienne pas compte de faits avérés, qu'on invoque à tort un réel déformé, qu'on impose un excès de sens ou un sens erroné aux phénomènes étudiés, qu'on n'apporte aucun élément solide à l'appui de ses dires... Ce sont des reproches que nous faisons tous, chaque fois que nous critiquons d'un point de vue empirique des propos tenus par un chercheur qui nous apparaît à cet égard, quel que soit son talent rhétorique, comme imprudent, léger voire incompétent. Dans les débats et les commentaires qui agitent en permanence notre tout petit monde de chercheurs, tout n'est pas qu'affrontements de paradigmes, antagonismes d'écoles et querelles de personnes : on dispute aussi de la véridicité des arguments produits, et l'on y met souvent en doute la teneur et la valeur empiriques, explicites ou latentes, des propos. Les « faits » ont beau être construits, ne pas exister en l'état, ne pouvoir être "récoltés", être indissociables du regard de l'observateur, qui parmi nous n'a jamais opposé « les faits » à des interprétations abusives et donné raison aux « faits $^{10} »$ ?

\section{Le ciseau surinterprétatif}

Pour comprendre d'où nous vient cette impression récurrente de sens « forcés » sur les données (ou de données «forcées » sur leurs réalités de référence), la métaphore du " ciseau surinterprétatif " peut ici être utile. La projection excessive de préconceptions d'un côté et la paresse méthodologique de l'autre fonctionnent en effet comme les deux branches d'un ciseau.

Les préconceptions, comme chacun sait, on essaie sans cesse d'y échapper (à coup de rupture épistémologique pour certains, par de plus simples exercices pour d'autres) sans jamais y arriver vraiment, puisqu'il n'est pas de donnée produite sans qu'elle réponde à des questions qu'on se pose, et qu'on ne peut se poser de question sans qu'il n'y ait une préconception à l'œuvre. Mais on conviendra qu'un peu de préconceptions ne veut pas 
dire trop. Ce n'est pas parce qu'il n'existe aucun compteur à repérer les excès de préconceptions que ceux-ci n'existent pas. Rien n'est en effet plus tentant pour un chercheur que de peindre la réalité aux couleurs qui lui conviennent, ou de prendre ses désirs scientifiques pour des réalités. Les incitations à l'excès sont multiples. Paradigmes (durs ou mous), postures heuristiques, théories locales ou de moyenne portée, idéologies de tous ordres, fantaisies idiosyncrasiques : ces éléments incontournables du paysage de toute recherche ne sont pas avares de préconceptions, plus ou moins productives, et il est souvent plus commode de les projeter sur la réalité sociale étudiée plutôt que de les mettre à l'épreuve de celle-ci.

D'un autre côté, les excès de préconceptions sont à l'évidence d'autant plus faciles à commettre qu'on baisse sa garde méthodologique. La vigilance méthodologique (qui n'a évidemment rien à voir avec le simple respect de procédures statistiques, et vaut autant pour le «qualitatif » que pour le «quantitatif ») permet justement de dresser quelques garde-fous contre la propension aux excès interprétatifs : le recoupement des sources, la recherche des contre-exemples, l'identification des propos, la compétence linguistique, et bien d'autres "tours de métiers ", aident à garder une souhaitable prudence empiriciste au sein même de la nécessaire prise de risque interprétative.

C'est donc cet intervalle, plus ou moins ouvert, entre les deux branches du ciseau, qui constitue l'espace de la surinterprétation, en tout cas dans la définition qui a été ici proposée de ce terme. En ce qui concerne nos disciplines, qui nierait que le jeté de présupposés sur terrain d'enquête ne soit un sport assidûment pratiqué? Qui ne remarquerait la langueur méthodologique et la désinvolture empirique dont certains sont affligés ? Sans chercher la paille dans l'œil d'autrui, n'avons-nous pas tous, dans de rares éclairs de lucidité, perçu que nous nous laissions parfois aller à d'excessives et séduisantes extrapolations facilitées par une vigilance empirique émoussée? La surinterprétation existe donc, bien qu'elle ne se laisse ni mesurer, ni réduire à une quelconque formule satanique. M'autorisera-t-on à en décrire quelques figures proéminentes et récurrentes?

\section{Figure 1 : la réduction à un facteur unique}

Un conflit divise le village A, charmante bourgade biélo-ukrainienne. Il oppose le maire à son premier adjoint. Toute la vie du village en est empoisonnée. Un anthropologue distingué s'intéresse à ce village, et à ce conflit. Il analyse sans hésiter toute l'affaire comme l'expression d'antagonismes « ethniques ». En effet le maire est de l'« ethnie » E1, et le premier adjoint de l'« ethnie » E2. Il y a déjà eu des tensions, des injures, des rumeurs, portant sur l'appartenance « ethnique » des uns et des autres. L'anthropologue en fait état pour appuyer son interprétation. Il ne manque pas non plus de citer une abondante littérature d'inspiration ethnologique sur les conflits séculaires entre les E1 et les E2, avec parfois intervention des E3. Mais il se trouve que le maire est aussi le beau-père de son adjoint (les mariages interethniques sont à A aussi fréquents qu'ils l'étaient en ex-Yougoslavie et au Rwanda). Et il y a bien sûr aussi beaucoup d'exemples de conflits entre beaux-pères et gendres dans la région de $\mathrm{A}$ (comme à Lyon).

Par ailleurs, le maire habite le quartier haut, et l'adjoint le quartier bas. L'histoire du village n'est pas avare de conflits entre les deux quartiers, dont chacun est 
multiethnique. De plus le maire est baptiste, et son adjoint s'est récemment converti à une nouvelle église évangélique. Là encore, on a pu constater que dans le pays la coexistence de ces deux confessions est loin d'être toujours pacifique.

Enfin, le maire a un caractère impossible, et le premier adjoint n'est pas commode. Tous deux sont de fortes personnalités, et se sont constitué leur propre réseau d'amis, de clients, de relations. Ces deux réseaux supportent d'ailleurs chacun un club de football différent. Ce n'est pas vraiment la grande fraternisation entre les deux réseaux.

Aussi trivial ou caricatural que soit cet exemple fictif, il renvoie à une pratique assez habituelle dans nos disciplines : réduire $n$ facteurs, empiriquement observables et pouvant tous jouer un rôle dans l'«explication » d'une situation sociale locale, à un seul d'entre eux. Tous les autres disparaissent comme par enchantement. Cette pratique n'est pas sans rapport avec une propension préalable, préprogrammée en quelque sorte, de celui qui s'y adonne à privilégier justement ce facteur-là, où que ce soit. On a ainsi les spécialistes de l'interprétation par l'ethnie, par la classe sociale, par le "genre », par l'appartenance religieuse, etc. Bien sûr ces différents facteurs, ou d'autres, peuvent jouer. Mais la complexité de la vie sociale et de ses interactions rend finalement rares les contextes où un facteur et un seul peut être invoqué. Et pourtant la "monofactorialisation", autrement dit le fait de ne proposer à un processus social donné qu'une seule « cause » ou " origine » là où l'expérience de la recherche comme l'histoire des sciences sociales devraient inciter à plaider la multifactorialité, est loin d'avoir disparu.

Rappelons aussi qu'il n'y a pas nécessairement obsession monofactorielle préalablement constituée, et qu'une recherche qui veut résolument privilégier l'empiricisme peut succomber elle aussi à la tentation de se focaliser sur un seul facteur, le plus apparent localement, ou le plus élégant scientifiquement, ou le plus séduisant intellectuellement, quitte à « oublier » tous les autres.

Certes, la mise en évidence d'une cause ultime, ou principale (" en dernière instance ", disions-nous autrefois en suivant Althusser) n'est en rien une ambition illégitime des sciences sociales, confrontées à une multiplicité de variables qui peut conduire à la démission interprétative. Une simple énumération ne prend pas de risques : mais y verrat-on de la bonne anthropologie (sociologie, histoire...) ? Elle doit bien déboucher un jour ou l'autre sur une tentative de pondération des facteurs. C'est un risque normal à prendre que de proposer que tel facteur soit considéré comme "dominant ». Mais cela ne saurait se faire au prix d'une impasse sur tous les autres. Dire « ce conflit est ethnique » (en ne fournissant que des "preuves » allant dans ce sens et en étant muet sur tout le reste) n'est pas la même chose que de dire « parmi tous les facteurs qui peuvent expliquer ce conflit et l'on détaille ces facteurs en fournissant les «preuves » correspondantes -,je pense que le plus influent est le facteur "ethnique", et voici pourquoi... » Nous sommes à un de ces lieux de "passage », aussi fluctuants que les Lettres de l'Atlantique, où l'on quitte le risque interprétatif légitime pour une surinterprétation qui s'auto-affranchit de contraintes empiriques. 


\section{Figure 2 : l'obsession de la cohérence}

Le célèbre ethnologue $\mathrm{V}$ a peint un tableau considéré comme achevé de la complexité du système cosmogonique, mythologique, rituel et symbolique des $P$. C'est d'ailleurs son chef-d'œuvre. On y voit que, au même titre que les Grecs, les Hébreux ou les Égyptiens, voire mieux qu'eux, les P, peuplade injustement méconnue de l'Océanie centrale continentale, avaient développé une cosmogonie particulièrement complexe, dont l'élaboration du système de correspondance ne laisse pas d'étonner. À chaque principe spirituel, révélé au fil des siècles par les Ancêtres, selon les dires des Grands Mythes, répond un élément rituel, repérable dans la grande diversité des cérémonies lignagères et villageoises qui ponctuent l'année, lequel renvoie à son tour à une plante, laquelle prend place dans un système de classification botanique où l'on reconnaîtra, au prix de quelques opérations routinières d'inversion, de transformation et de substitution, un décalque du système cosmogonique précédemment identifié. Qui plus est, on retrouvera dans l'habitat, les travaux artisanaux, et les préparations culinaires, pour ne citer que ces quelques activités, les traces de cette matrice symbolique omniprésente.

La mariée est souvent trop belle. Pour qui a été sans cesse confronté à des savoirs oraux hétérogènes, fluctuants, voire "en miettes", inégalement répartis, diversement structurés, peu systématisés, stratégiquement proférés, politiquement manipulés, les fresques symboliques grandioses ou les tableaux «ethno-scientifiques» bien ordonnés laissent rêveur, et sceptique ${ }^{11}$. L'ordinaire de l'anthropologie, en tout cas de l'anthropologie de terrain, c'est quand même l'ambiguïté et la multiplicité des systèmes de valeurs, les contradictions et les divergences incessantes dans les propos, la structure floue et les limites incertaines des "systèmes de représentations ${ }^{12}$ ", la discordance fréquente entre normes et pratiques...

Certes il nous appartient de mettre autant d'ordre qu'il se peut dans ce désordre, ce fouillis, cet enchevêtrement. Par définition, les sciences sociales, en créant du sens, vont produire un minimum de cohérence : il n'est pas de sens dans l'incohérence. Mais cette exigence de cohérence inhérente à l'activité intellectuelle même de nos disciplines ne signifie pas pour autant carte blanche accordée à la "cohérentisation" ou à la "systématisation » tous azimuts. L'imagination " cohérentisante » et «systématisante » débridée dont l'anthropologie a parfois fait preuve n'est pas ce dont elle a le plus à s'enorgueillir ${ }^{13}$. L'exercice comparatif, qui, comme chacun sait, est au principe même des sciences sociales, est plus productif lorsqu'il s'appuie sur les divergences que lorsqu'il ne s'intéresse qu'aux similitudes. L'exercice monographique lui-même, indissociable de toute ambition comparative, gagne à s'interroger sur les ambiguïtés, les contradictions, les différences plutôt qu'à les aplatir ${ }^{14}$.

\section{Figure 3 : l'inadéquation significative}

Imaginez un anthropologue poldave, ne parlant quasiment pas le français, débarquant à Marseille, fort désireux de confirmer au plus vite la déjà flatteuse réputation de chercheur brillant et audacieux qu'on lui avait faite à l'université de $\mathrm{Q}$. 
(capitale intellectuelle du pays). Au bout de quelques jours, il avait déjà repéré que tous les aborigènes étaient désignés par le terme d'adresse " cong ", manifestement associé à une appartenance clanique, et qu'un sous-lignage pouvait être distingué à partir de l'expression « niktamèr ». Un mois plus tard, il avait considérablement avancé dans la reconstruction du système religieux local, à base de totems et de grisgris pieusement déposés devant l'autel d'une divinité païenne (sans doute l'ancêtre maternelle des autochtones), connue sous divers avatars (bonmèr, saintvierj, notdam), qui exige de ses fidèles qu'ils entretiennent nuit et jour des feux en cire devant son tombeau. Après six mois, devenu, croyait-il, expert en français, il se sentait capable d'analyses sémiologiques plus fines encore : ainsi avait-il mis en valeur, par l'enregistrement patient des conversations quotidiennes, que les indigènes croyaient tous en trois forces mystiques distinctes, mais associées par des liens fort complexes qu'il entendait bien démêler ultérieurement s'il obtenait pour ce faire une bourse Fulbright, forces appelées respectivement «bol », «po » et « shans », que l'on rendait responsables des heurs et malheurs incessants de l'existence : jai-pad-bol, mank-d-po, la-shans-séfett-lamal, étaient autant de plaintes rituelles adressées à ces forces lorsqu'elles avaient abandonné l'intéressé, plaintes débouchant le plus souvent sur des formules propitiatoires du type «skusi-sava-marshé».

J'ai tenté, en recourant à ce procédé rhétorique désormais classique en anthropologie ${ }^{15}$, de montrer simplement à quel point la " fidélité » aux conceptions locales et à l'univers de sens des autochtones est difficile à restituer pour l'observateur étranger dès lors qu'il ne maitrise pas les codes locaux. C'est certes un piège qui attend évidemment plus l'ethnologue travaillant en pays lointain, voire l'historien, que le sociologue (quoique parfois...). Qui ne pourrait mettre divers noms derrière des méprises illustres ou obscures quant aux significations "indigènes»? On voit mal comment, sur la base de reconstitutions, de descriptions ou de traductions qui « manquent " gravement la réalité de référence, on peut proposer des interprétations ayant un quelconque indice de véridicité. Rien n'empêche néanmoins ces interprétations "empiriquement fausses " d'être, pour les lecteurs non avertis de la réalité locale, parfaitement séduisantes.

Pourtant, l'«émicité », ou encore le respect de l'«adéquation significative », pour reprendre un terme d'origine weberienne, autrement dit la capacité des sciences sociales à restituer autant que possible les systèmes de sens des groupes étudiés, à rendre compte des valeurs, des codes et des normes autochtones, à recueillir les représentations propres aux acteurs sociaux, apparaissent comme des conditions nécessaires de toute recherche empirique et de tout travail interprétatif. Certes, ce ne sont pas des conditions suffisantes. Par exemple, le recours à d'autres types de données ou à d'autres registres d'« adéquation " ('"« adéquation causale », en suivant Weber, ou, dans un autre langage, les données "étiques») s'impose. Mais des propositions interprétatives qui seraient « inadéquates significativement », qui reposeraient sur des faux-sens ou des contresens au niveau "émique », perdraient toute validité. En fait l'inadéquation significative est si vaste en ses manifestations qu'elle peut être décomposée en plusieurs sous-figures.

\section{Sous-figure 1 : l'incompétence linguistique}

Cette incompétence peut-elle sévir même lorsqu'on travaille dans sa propre langue mais sur d'autres parlers (le verlan ou le marseillais pour un intellectuel parisien) ? Je ne sais, 
mais elle est évidemment beaucoup plus menaçante lorsqu'on travaille dans une langue étrangère, et plus encore lorsque cette langue étrangère est très éloignée culturellement et linguistiquement (cas des langues orales d'Afrique ou d'Océanie, par exemple). La nonmaîtrise des langues locales en situation quotidienne, travers déplorable opposé au principe même de toute bonne ethnographie, mais hélas particulièrement répandu dans l'anthropologie française, pave évidemment le chemin aux étymologies fantaisistes, aux rapprochements homophoniques non fondés, aux définitions partielles et partiales des termes locaux, aux délires interprétatifs à base d'erreurs sémiologiques ${ }^{16}$.

\section{Sous-figure 2 : la traduction orientée}

Celle-ci se faufile au sein de la difficulté bien réelle à laquelle on est confronté lorsqu'on veut traduire en français (en anglais, en espagnol...) un terme local dont le champ sémantique n'a en fait pas d'équivalent possible dans les grandes langues occidentales ${ }^{17}$. Prenons un exemple simple. Les spécialistes des séances de possession, responsables des « confréries » de médiums, sont appelés serkin bori en haoussa, zimma en songhay-zarma, etc. Comment va-t-on les désigner en français, langue où ce type de fonction n'existe ni de près ni de loin? Les deux traductions de loin les plus fréquentes sont "prêtres » ou " guérisseurs ». Voilà le biais « religieux » d'un côté, le biais « thérapeutique » de l'autre ${ }^{18}$. Certes on ne peut échapper à ce genre de dilemmes, sauf à parsemer ses ouvrages de termes locaux non traduits, les rendant du coup quasi illisibles. Mais, du moins, peut-on ne pas s'engouffrer aveuglément dans l'avenue sémantique ouverte par le biais en question et mettre en garde le lecteur, quand on est obligé de recourir à une traduction forcée, sur les risques de l'opération.

\section{Sous-figure 3 : le « durcissement »}

Toujours dans une problématique de la traduction, il s'agit cette fois d'un procédé qui «sort » une expression autochtone de ses contextes de profération, souvent machinaux, distraits, ambigus, pour la « surcharger » d'exégèse (volontiers symbolique). Reprenons l'analyse de Keesing (qui lui-même s'appuie sur Lakoff et Johnson) ${ }^{19}$ : les métaphores conventionnelles relèvent d'une appréhension lâche et routinière du monde, à partir d'images évoquant des perceptions et des sensations sans prétention explicative aucune, du type «j'ai l'estomac dans les talons», ou «la lune est pleine». Transformer une métaphore conventionnelle en un énoncé métaphysique ou en une assertion spéculative, c'est produire un énorme contresens. On risque ainsi, si l'on n'y prête garde - et les exemples en ethnologie exotique sont hélas nombreux -, de détecter une parcelle de "vision du monde ", de "conception de la personne ", voire de cosmogonie, à l'œuvre dans les banales expressions ou locutions «toutes faites" qui parsèment les discours quotidiens, équivalent chez nous de «le soleil se lève à 5 heures " (ceci témoignerait d'une résistance massive à la modernité scientifique, au nom de l'héritage chrétien prégaliléen...), ou de " son cœur est brisé » (les conceptions indigènes considèrent l'organe du cœur comme le siège des sentiments). 


\section{Sous-figure 4 : l'imputation émique abusive}

Cette sous-figure de l'inadéquation significative est particulièrement vaste, puisqu'elle couvre toutes les situations où le chercheur attribue aux « indigènes » des motivations, des comportements ou des logiques qui apparaissent en fait, pour un observateur mieux informé ou plus attentif, comme en contradiction avec tout ce que l'on peut savoir des motivations, comportements et logiques desdits « indigènes ». L'histoire et la littérature connaissent bien de tels anachronismes psychologiques ou culturels, mais l'anthropologie a aussi son lot d'analogues ethnocentrismes.

Tout ceci n'est certes pas facile à « démontrer » puisqu'il faut en général passer par des indicateurs indirects et complexes pour tenter de réfuter les imputations abusives. Elles n'en existent pas moins. Pour prendre un exemple éculé, combien de raisonnements ne sont-ils pas basés sur l'attribution à l'acteur d'une rationalité unique de maximisation des profits et de minimisation des coûts, alors que la psychologie cognitive témoigne de ce que les processus de prise de décision sont autres, et que la socio-anthropologie montre que les logiques d'action sont à la fois diverses et contraintes culturellement?

41 Le « résistocentrisme » relève lui aussi de l'imputation émique abusive. On transformera ainsi tout paysan (ou, selon les besoins, tout jeune, toute femme, tout ouvrier) en "résistant», actif ou passif, direct ou indirect, manifeste ou latent, par une lecture systématiquement orientée du moindre de ses comportements. Par leurs rituels, par leurs rumeurs, par leurs jeux et leurs beuveries, par leurs migrations, ils expriment leur résistance. Par leur refus des migrations, ils expriment encore leur résistance ${ }^{20}$.

L'«hypersymbolisme », transformant les «indigènes » en théologiens, mythologues, mystiques ou philosophes à plein-temps, est du même ordre.

3 Les exemples pourraient être énumérés à l'infini. Dans tous les cas, on a affaire à un cas particulier de la projection de préconceptions, qui consiste à affubler les « autochtones » des vêtements qu'on leur trouve seyants.

\section{Figure 4 : la généralisation abusive}

W était incontestablement un grand érudit. Bien qu'analphabète, il avait écouté dès son jeune âge les prêches des imams et des curés dans sa petite ville natale, il avait suivi avec grande attention et pendant des années les séances de géomancie de son vieil oncle devin, il avait mémorisé les dires des griots qui déclamaient les hauts faits des princes d'antan au cours des veillées, il ne manquait jamais non plus d'être à l'écoute sur son transistor pour les émissions historiques de RFI. J'allais oublier : une respectable carrière de tirailleur sénégalais l'avait mené de Fréjus à Saigon, de Bordeaux à Constantine ; une seconde carrière de commerçant ambulant lui avait fait connaître Dakar, Bamako et Douala. Un jour vint l'ethnologue. Ils sympathisèrent. L'ethnologue fut ébloui par les connaissances de W. L'ethnologue travaillait sur « la conception du monde et de la personne chez les H ». Bien sûr W était lui-même $\mathrm{H}$ (sinon ils n'auraient pas ainsi sympathisé). C'était un des $\mathrm{H}$ les plus vieux et les plus respectés. Six ans plus tard (délai normal dans la profession), la thèse de l'ethnologue était soutenue. On se doute que les propos de $\mathrm{W}$ en formaient l'ossature. Trois ans plus tard (là, l'ethnologue avait fait nettement plus vite que la 
moyenne), un livre paraissait, qui s'attaquait au difficile problème de l'unité culturelle de l'Afrique : y a-t-il une conception africaine de la personne?

(l'ethnologue avait laissé tomber les histoires de conception du monde, qui désormais l'ennuyaient). Suivant en cela l'opinion de W, mais en l'étayant de nombreuses lectures érudites, l'ethnologue disait que oui.

C'est à tous les niveaux et dans tous les domaines qu'intervient la généralisation abusive. Distinguons, par commodité, généralisation interne et généralisation comparative. La généralisation abusive peut d'abord être "interne " à un terrain. C'est tout le problème par exemple des « représentations partagées » ou des «significations partagées » (shared meanings) $)^{21}$. Jusqu'à quel point les propos d'un « informateur " peuvent-ils être tenus pour «représentatifs » d'un groupe ou d'une culture? Faute de vigilance méthodologique (recoupements, triangulation, saturation...) le collaborateur privilégié de l'anthropologue devient vite son "grand témoin ", sa source quasi unique de données, son inspirateur. C'est tout le problème aussi de l'unité d'analyse du chercheur: en quoi une famille d'accueil exprime-t-elle la situation plus générale des autres familles locales, en quoi un site d'enquête villageois renvoie-t-il à l'ensemble des villages d'une région, en quoi une cité HLM est-elle l'archétype de toutes les cités HLM du pays? Quel degré de généralisation doit-on accorder à des propos fondés sur des données "qualitatives " toujours fortement localisées et circonscrites dans leur production, qui ne constituent en quelque sorte que quelques points dans l'espace social de référence dont le chercheur entend rendre compte? Des travaux anthropologiques sur «les jeunes des banlieues parisiennes », ou "l'amour chez les Peuls de l'Oudalen » ne sont évidemment fondés le plus souvent que sur quelques entretiens avec quelques jeunes de quelques banlieues, ou une observation plus ou moins participante dans quelques campements peuls.

Mais n'est-il pas abusif de parler de surinterprétation, là où on a affaire à des phénomènes d'extrapolation inévitable, que le chercheur peut tenter de garder sous contrôle ? La réponse est, encore une fois, "oui mais quand même... ». L'extrapolation étant inévitable, et nécessaire, elle doit se faire avec d'autant plus de précaution, de prudence et de vigilance. Or, trop souvent, précaution, prudence et vigilance ne sont pas du voyage. Mais il est vrai que le manquement aux exigences de véridicité empirique est dans le cas de la généralisation interne abusive particulièrement difficile à plaider « données en main ».

Il l'est moins dans le cas de la généralisation abusive externe, à savoir l'extension comparative indue, où la désinvolture se fait plus évidente. Ce que j'ai dit sur un groupe $\mathrm{X}$ (à supposer que les généralisations internes à ce groupe $\mathrm{X}$ soient fondées) et que j'applique sans hésitation à l'ensemble $\mathrm{Y}$, dont le groupe $\mathrm{X}$ peut être considéré comme un sous-ensemble, peut être pris en défaut dès lors que je ne me donne pas la peine d'argumenter empiriquement cette extension.

On a chez Clastres, cela a déjà été fort bien relevé par d'autres ${ }^{22}$, un bel exemple de généralisation abusive par extension comparative. Ce qu'il a «démontré » à propos des Indiens Guayaki ou Tupi-Guarani (si tant est que la démonstration soit valable et empiriquement fondée, ce qui est une autre histoire ; mais laissons-lui ici le bénéfice du doute) est étendu par lui à l'ensemble des sociétés amazoniennes, voire indiennes, puis, tant qu'à faire, à toutes les sociétés " primitives ».

Toutes les formes du «grand partage » relèvent d'ailleurs de la généralisation abusive. Mais on pourrait aussi se demander si le «modèle mécanique » de Lévi-Strauss ${ }^{23}$ ne prête 
pas le flanc au soupçon, et ne constitue pas à certains égards une incitation à la généralisation abusive. Certes, il s'agit, contrairement au " modèle statistique ", de rester proche des phénomènes, des cas, on dirait aujourd'hui des acteurs. Mais c'est souvent pour sauter directement vers l'universel, le renoncement à la représentativité statistique semblant parfois autoriser la modélisation « mécanique » à se libérer de tout garde-fou en matière de comparatisme raisonné. L'anthropologie ne s'est-elle pas fait une spécialité du "saut interprétatif " sans filet, autrement dit du raccourci entre singulier et général ? Nous sommes une fois de plus confrontés au problème épineux du "passage » à la surinterprétation. Si les amarres empiriques sont rompues, que ce soit au niveau de la validité du modèle quant à sa situation empirique de référence ou que ce soit au niveau de l'extension du modèle par l'absence de contrôle sur sa pertinence dans d'autres situations empiriques, n'y a-t-il pas suspicion légitime de surinterprétation ${ }^{24}$ ?

\section{Figure 5 : le coup du sens caché}

Il était une fois un club de rugby provincial qui coulait une vie tranquille en division d'honneur. Une équipe de chercheurs en anthropologie du sport vint à passer par là, et décida de s'intéresser à ce bonheur paisible d'une équipe sans histoire. La subvention municipale tombait chaque année, l'équipe assurait son maintien en milieu de tableau, les troisièmes mi-temps étaient aussi chaleureuses que bien arrosées, le président ignorait la liaison que le capitaine de l'équipe avait avec son épouse. Tout allait bien. Patatras! L'enquête était déjà bien avancée, quand un beau jour tout se dérégla. L'équipe commença une série de douze défaites qui devaient la mener en relégation, une beuverie anodine se termina par une intoxication alimentaire collective due à une pizza douteuse amenée par le maire, le président du club eut la douleur de prendre les fautifs en flagrant délit, etc.

Dans l'équipe de chercheurs deux interprétations s'opposèrent (qui ont d'ailleurs amené ultérieurement l'éclatement du laboratoire). Pour les uns, une durée excessive de tranquillité ne pouvait qu'aboutir à un tel dérèglement, par une inversion des contraires typiques de l'inconscient collectif des petits groupes : les pulsions de mort trop longtemps refoulées avaient provoqué un regrettable effondrement des moi et des surmoi au profit des ça. Pour les autres, spécialistes en ethno-occultisme, un sort avait été jeté par la femme du maire, elle aussi amoureuse du capitaine de l'équipe.

Bien sûr, on ne trouvera jamais d'interprétations aussi grotesques émanant de chercheurs sérieux (du moins dans ce registre et sur cette équipe-là). Mais le principe du «sens caché », invérifiable de quelque façon que ce soit, pour « rendre compte » d'une situation sociale, a beaucoup d'amateurs et non des moindres.

Par définition, en quelque sorte, on se libère de toute plausibilité empiriquement fondée, émique ou étique, puisque le sens proposé est "opaque » aux acteurs (pas d'adéquation ou d'inadéquation significative) et qu'aucune procédure d'observation ne peut non plus confirmer ou infirmer l'hypothèse. Ce type de surinterprétation est autiste, autoréférentiel. Il marche en boucle, et marche toujours, puisque l'invocation d'une "réalité cachée » suffit à fonder l'argumentation, sans craindre de démentis issus de données quelconques. Multipliez les contre-exemples, vous ne pouvez déstabiliser l'argumentation. Le crime est presque parfait; il ne l'est pas tout à fait en ce que 
justement l'absence de toute référence empirique mobilisable signale le coupable, si tant est qu'on veuille y prêter attention. Une théorie qui se donne pour empirique (ne seraitce qu'en se réclamant de l'anthropologie, de la sociologie ou de l'histoire) mais qui ne se déploie qu'en dehors de toute empiricité, par l'invocation de "principes» ou de « logiques » en elles-mêmes invérifiables ou inexplorables quasiment par définition, voilà qui devrait mettre la puce à l'oreille.

51 Et pourtant le «coup du sens caché » continue à avoir du succès, mais avec de nombreuses variations et transformations. L'inconscient, par exemple, se porte très bien, alors que d'autres modèles sont devenus obsolètes. Le « sens de l'histoire » ne fait plus un malheur, et le "fonctionnalisme ", qui relève bel et bien de ce genre, est lui aussi plutôt dévalué. Une variante toujours fort en usage est l'attribution à des ensembles abstraits (agrégats sociaux ou institutions, concepts, construits idéologiques, artefacts scientifiques) de qualités de volition, de décision, d'intentionnalité, proprement humaines. «Le système veut que... ", « pour se reproduire la culture a besoin de... », «le monothéisme a pour objectif de...», "conformément aux attentes de la classe laborieuse... », «il est dans la nature de l'habitus de permettre... », etc. C'est le problème du «macranthrope », fiction de contrebande dont les méfaits ont déjà été soulignés par Mannheim ${ }^{25}$. On voit ici une des dérives surinterprétatives favorites du "holisme», de même qu'on a vu plus haut que, dans la figure de l'imputation abusive, pouvait se glisser une des dérives surinterprétatives favorites de l'individualisme méthodologique.

\section{La combinaison des figures}

Toutes ces figures (et on pourrait sans doute en trouver quelques autres ${ }^{26}$ ) se cumulent bien sûr volontiers, à deux, à trois, voire plus.

Prenons l'exemple de l'Essai sur le don ${ }^{27}$. Le succès international non démenti du mana et du hau doit évidemment beaucoup au talent de Mauss, mais il n'est pas non plus sans liens avec les idéologies, scientifiques ou non, qui, de Bataille à Deleuze, accréditent une version du "grand partage» qui voit dans la version océano-maussienne du don l'antithèse de la vénalité capitaliste. Ces "préconceptions» en ont "rajouté" en surinterprétation, mais le travail de Mauss lui-même, vu d'aujourd'hui, est loin d'en être exempt. Ce qui est apparu longtemps comme empiriquement fondé semble aujourd'hui non seulement dé-crédibilisé ou dé-plausibilisé aux yeux des spécialistes de l'Océanie, mais aussi, sur certains points, quasi réfuté. On sait maintenant que, en Polynésie ou en Mélanésie, le mana n'était ni une substance, ni l'âme du donateur enfermée dans la chose donnée, ni un concept métaphysique, mais une notion populaire évoquant "efficacité ", "pouvoir ", "capacité ", et renvoyant aux ancêtres et aux esprits ${ }^{28}$. Il en est de même pour le hau, même si, de Firth à Sahlins, on débat encore de la meilleure traduction du texte maori à partir duquel Mauss a développé son argumentation ${ }^{29}$. Il est d'autant plus intéressant de constater l'écart qui sépare certaines critiques contemporaines des critiques anciennes d'un Lévi-Strauss. Celui-ci, en effet, se souciait fort peu en 1950 de questionner la validité des matériaux utilisés par Mauss ${ }^{30}$. Bien plus, il accusait Mauss (certes avec déférence) de s'être fait mystifier par les conceptions indigènes en les reprenant trop à son compte... Sa critique était donc purement "interprétative», ou plutôt «réinterprétative», puisqu'il s'agissait de remplacer l'analyse quelque peu « substantiviste » de Mauss par une analyse « relationnelle », autant dire structuraliste. De nos jours, si les commentaires purement réinterprétatifs n'ont pas disparu ${ }^{31}$, ils 
côtoient désormais des critiques fondées sur une remise en question des sources de Mauss et de l'usage qu'il en a fait (ceci allant des erreurs de traduction à la non-prise en compte des conditions d'énonciation des récits utilisés) ainsi que sur un recours à des données nouvelles, inconnues à l'époque.

De ce fait, quel que soit l'hommage qu'on doive rendre à Mauss pour son souci proclamé de s'appuyer sur les représentations " indigènes » et d'y fonder son comparatisme, force est de constater que c'est bien le contraire qui s'est passé, puisqu'on peut relever dans l' Essai sur le don le cumul de la plupart des figures de la surinterprétation. L'erreur de traduction assortie à un durcissement de la métaphore (figure de l'inadéquation significative) semble claire. Mais la généralisation abusive s'y mêle allègrement, qui étend l'acception erronée de mana ou de hau aux « sociétés primitives » dans leur ensemble. L' obsession de la cohérence est aussi passée par là, en transformant le mana en principe général de la réciprocité, voire en «vision du monde » tous azimuts. Enfin la projection d'un ensemble complexe de transactions sur sa seule signification «mana » n'est-elle pas de l'ordre de la réduction à un facteur unique?

Certes on ne peut parler à ce sujet de surinterprétation que par anachronisme, en ce que rien de tout cela n'était perceptible à l'époque de Mauss. Le non-spécialiste de l'aire n'y voit d'ailleurs aujourd'hui encore que du feu. Mais la différence est qu'il existe justement désormais des spécialistes de l'aire qui peuvent démentir les données de seconde main sur lesquelles Mauss a travaillé. Le niveau d'exigence empirique s'est de ce point de vue-là considérablement élevé, au moins là où des compétences en véridicité peuvent se manifester. L'avantage d'un débat sur la surinterprétation est peut-être d'attirer l'attention sur ceci, en appelant de ce fait à plus de vigilance tous ceux qui utilisent machinalement des références (classiques ou à la mode) extérieures à leurs domaines de compétence (autant dire nous tous).

Mais il est vrai aussi que, dans le système de références érudites des sciences sociales, certaines œuvres, devenues "classiques", ne fonctionnent plus que comme de purs "textes", décrochés de fait de leurs référents empiriques. L'anthropologie (comme la sociologie ou l'histoire) a ainsi une composante « histoire des idées » où les livres réputés qui y ont pris place figurent, non en raison de leur pertinence empirique (souvent mise à mal par des travaux ultérieurs plus modestes mais mieux documentés), mais du fait de la nouveauté, de la séduction ou de l'originalité "en leur temps" des "thèses", des " paradigmes », des " postures heuristiques ", et parfois des formules qui y figurent et qui leur ont valu la notoriété. Certes, comme toutes les œuvres se réclamant des sciences sociales, ces travaux savants incorporaient originellement une légitimation empirique. Mais tout se passe comme si celle-ci s'était peu à peu dématérialisée et estompée pour ne plus laisser place qu'au seul brio argumentaire ou au caractère emblématique de la démonstration théorique. On peut aujourd'hui à peu près prouver que l'Essai sur le don est une œuvre empiriquement non fondée, ou que Le suicide de Durkheim recourt à une méthodologie contestable; mais cela ne menace plus la place de ces références illustres dans l'histoire de l'anthropologie ou de la sociologie. De par ce délestage empirique, elles prennent place dans un circuit herméneutique savant (ou méta-interprétatif) dans lequel on ne leur demande plus de comptes quant à leur véridicité32.

Certes, il ne serait guère convenable de reprocher aux ancêtres totémiques de ne pas avoir respecté par anticipation la vigilance empirique qu'on est en droit d'exiger désormais de nos collègues et de nous-mêmes. Mais on peut déplorer, sans en rien porter atteinte à leur mémoire, que les références massives à leurs écrits ne soient pas plus 
souvent assorties de mises en garde quant au caractère obsolète des procédures et au manque de fiabilité des données. Car il ne faut pas se le cacher : c'est l'invocation de ces prédécesseurs illustres qui permet souvent de justifier chez certains de nos contemporains leurs propres excès surinterprétatifs...

\section{Le nécessaire risque interprétatif}

58 Ces diverses figures de la surinterprétation que nous avons passées en revue ont ici un évident caractère de "repoussoir ». Je n'entendais pas, en effet, dissimuler une position épistémologiquement normative: la surinterprétation est une des plaies de notre profession. Il ne faudrait pas pour autant croire qu'une attitude frileuse, arc-boutée au plus près des données, et minimisant l'interprétation, soit la meilleure façon de se prémunir de la tentation surinterprétative. Nous avons déjà récusé par avance les tentations de la "sous-interprétation ». Qu'il soit clair que la prise de risque interprétatif empiriquement contrainte est au coeur de toute activité de recherche en anthropologie. En ce sens, le piège de la surinterprétation est sans cesse présent, car sans prise de risque interprétatif il n'est pas de sciences sociales. On sait à quel point la posture confortable qui consiste à dire seulement: « tout est beaucoup plus complexe que vous nous l'avez dit ", sans proposer de modèle alternatif, est improductive. On sait que l'attitude de critique permanente... des autres, au nom d'un purisme méthodologique qu'on serait bien en peine soi-même de respecter, est vaine. On sait que le repli sur une ethnographie purement descriptive et notariale est stérile.

Or les procédures de la nécessaire prise de risque interprétative peuvent sans cesse déraper vers la surinterprétation. On l'a vu dans notre exploration rapide des figures de cette dernière. Chacune d'entre elles était liée à un exercice normal, nécessaire, salutaire de l'imagination anthropologique (sociologique, historique). Pas d'anthropologie sans recherche de facteurs plus ou moins dominants ou de cohérence, sans traduction, sans généralisation, sans recherche de sens non "visibles». Mais cela ne signifie pas pour autant que l'on doive se résigner à la réduction à un facteur unique, à l'obsession de la cohérence, à l'inadéquation significative, à la généralisation abusive, au « coup du sens caché ».

60 Certains pourraient plaider en faveur d'un droit à la surinterprétation au nom de la nécessité de la prise de risque interprétatif et des bienfaits de l'imagination anthropologique. $Y$ aurait-il donc une surinterprétation féconde et une surinterprétation stérile, la première obligeant à tolérer la seconde, et ce d'autant plus qu'il est parfois difficile de faire le départ entre une intuition géniale et une fantaisie brillante ? Ce serait en fait se méprendre sur le sens qui a été ici donné à « surinterprétation ». Les prises de risques et les sauts interprétatifs raisonnés, dès lors qu'ils sont empiriquement argumentés, qu'ils ne rentrent pas en contradiction avec les données connues de l'époque, et qu'ils ne se prétendent pas plus étayés empiriquement qu'ils ne le sont en fait, ne peuvent être qualifiés de surinterprétations. C'est là, on y revient, l'ambiguïté même du terme de surinterprétation, qui pourrait faire croire qu'on a simplement affaire à un "plus » d'interprétation, à une interprétation en "sur-régime ", alors que c'est l'absence de vigilance méthodologique ou argumentative par rapport aux « données » qui «signe» pour nous la surinterprétation. Elle peut bien s'affubler des atours les plus "théoriques» et les plus prétentieux, ou se targuer d'une audace interprétative volontiers autoproclamée, la surinterprétation, dans l'acception ici utilisée, est, d'abord, 
un «déficit» en sens, en ce qu'elle «oublie » toutes les autres significations que les données qu'elle néglige ou déforme pourraient porter ou susciter. Autrement dit, la surinterprétation, toujours dans le sens que nous lui donnons, n'est ni du sens en plus, ni de l'audace en plus, en bien ou en mal, c'est du sens en moins et de l'audace en moins par rapport au travail empirique. C'est un moins-disant empirique. C'est un déficit en exigence scientifique, dans toutes ses dimensions : théoriques et hypothétiques, comme descriptives et factuelles. Succomber aux sirènes de la surinterprétation, c'est, en pliant les données à sa convenance, céder à la facilité, en refusant le défi qu'une prise en compte de la complexité de celles-ci adresse à l'imagination interprétative rigoureuse.

\section{La critique et le contre-exemple}

61 Au terme de ce parcours, on ne peut éluder la redoutable question du «comment faire?" compte tenu qu'il n'y a ni vaccin, ni antidote, ni préservatif. On ne voit guère qu'un indéfinissable mélange de "métier» et de "scrupule empiriste » qui puisse préserver des pièges les plus visibles de la surinterprétation, du fait qu'il n'y a ni règles formelles ni procédures méthodologiques dont le respect permettrait d'interpréter en toute sécurité. La véridicité étant intégrée aux démarches argumentatives et interprétatives et en étant indissociable, elle ne peut être garantie à l'extérieur d'elles, et a priori, par l'établissement d'une sorte de périmètre de sécurité méthodologique extrainterprétatif et extra-argumentaire. Et pourtant on ne peut renoncer à sa quête, ni à s'en prévaloir, ni à s'indigner lorsqu'elle fait défaut. D'où cette invocation au savoir-faire et à la vigilance, bien qu'un tel mélange soit difficilement définissable en termes épistémologiquement acceptables. Une pincée d'expérience professionnelle, une pincée de déontologie, cela ne fait peut-être pas très sérieux. D'autre part, en appeler à la " conscience méthodologique» de chacun peut laisser sceptique quant à l'efficacité de telles exhortations morales. Et pourtant... Peut-être peut-on quand même suggérer deux opérations intellectuelles dont l'exercice répété est ce qui existe encore de mieux tant en matière de prévention que de traitement.

62 La première est le débat, la critique, parfois la controverse. Dans nos disciplines, plus l'exercice de la pensée critique sera favorisé (institutionnellement, scientifiquement, intellectuellement), y compris au niveau du contrôle de la véridicité, mieux pourra être régulée la tension entre empiricité et interprétativité. La critique, à cet égard, doit aussi porter sur les "preuves", les "données produites", la plausibilité empirique, la véridicité des assertions, et non seulement sur les affrontements de paradigmes. Un grand progrès, à cet égard, réside, en anthropologie, dans la multiplication des « terrains revisités ». Le re-pâturage sinon le sur-pâturage anthropologique devient une réalité. Loin de le déplorer, au nom d'un exotisme de pacotille, il faut s'en réjouir. Il devient dès lors possible de procéder à des " contre-expertises ", à une "critique des sources " qui faisaient autrefois cruellement défaut avec le système de la chasse culturelle gardée. Le rapport empirie/interprétation peut devenir enfin objet de critiques documentées. La présomption de surinterprétation devient argumentable. C'est une sorte de révolution en anthropologie, dont on n'a guère souligné l'importance.

La seconde opération intellectuelle salutaire a pour nom " contre-exemple». C'est aussi une arme utilisée dans les débats. Mais ceux-ci, avec ou sans usage de contre-exemples, portent presque exclusivement sur des produits finis. Les retombées positives de la critique ex-post sont donc indirectes et différées. Le contre-exemple peut en revanche 
servir à toute étape du processus de recherche, et permettre de modifier celui-ci. Il a en effet le grand avantage d'être un argument empirique. D'un côté il oblige à retourner aux données, à ce double rapport "réalité »/données et données/interprétations que nous évoquions au début. Il donne chair, forme, contenu à l'exigence de contrôle méthodologique. D'un autre côté c'est une contre-interprétation latente, une incitation à l'interprétation alternative empiriquement mieux fondée ${ }^{33}$. Loin de nous tirer en arrière ou de nous faire tomber de la surinterprétation au renoncement interprétatif, il oblige à rebondir, en sommant l'imagination interprétative de produire un "modèle " plus exigeant en plausibilité empirique et en véridicité, car intégrant le(s) contre-exemple(s).

On remarquera que plus l'usage du contre-exemple intervient tôt dans le processus de recherche, plus il est efficace et permet d'améliorer la qualité interprétative. En fin de parcours, déjà incorporé à un débat voire une polémique, quand les points de vue sont déjà structurés, cristallisés et que les ego sont en jeu, on peut douter que la cible concernée en apprécie toute la portée et remette en cause un modèle interprétatif déjà clé en main. En début de parcours, quand on en est à l'étape de production des données (étape où les premières hypothèses interprétatives ne sont pas encore "durcies » et peuvent laisser encore place à d'autres, plus fécondes et plus empiriquement fondées), le contre-exemple est un puissant stimulant intellectuel et méthodologique.

Reste évidemment la question pratique et triviale: qui, sur le terrain, va dire à l'ethnologue : « ton hypothèse actuelle n'est pas satisfaisante, car il y a le cas D et le cas $G$ que tu as oubliés et qui la mettent en cause » ? L'exercice solitaire de l'anthropologie, qui reste dominant dans notre discipline, rend l'affaire difficile. Certes, une certaine forme de schizophrénie, dont d'ailleurs la profession n'est pas indemne, peut aider. Mais, en général, c'est bien ce mélange de savoir-faire et de vigilance, que l'expérience professionnelle permet d'acquérir (sinon chez tous, du moins chez beaucoup), qui va tant bien que mal permettre à l'anthropologue en pleine enquête de tenir un dialogue critique avec lui-même.

Il est une autre solution, toutefois, pour laquelle je voudrais plaider pour finir. C'est l'enquête à plusieurs. L'existence d'une équipe travaillant sur un terrain commun, sur la base bien sûr d'affinités problématiques et méthodologiques, permet de fonctionner sur un va-et-vient permanent entre hypothèses interprétatives et recherches de données vérifiant ou infirmant ces hypothèses, entre données imprévues et impertinentes et interprétations les organisant significativement. Un tel processus est d'autant plus efficace qu'il est collectif et que chacun peut opposer à tout moment des contre-exemples à chacun. Certes un mécanisme de surenchère interprétative peut aussi se mettre en place et, pas plus que toute autre procédure méthodologique, l'enquête collective n'est une garantie permanente et sûre contre la surinterprétation. Mais une certaine expérience de sa pratique m'incite à penser que, au moins dans certaines conditions, et en particulier lorsqu'elle se donne la libre critique mutuelle et la recherche de contreexemples comme règles $\mathrm{du}$ jeu, elle permet à la fois de stimuler l'imagination interprétative empiriquement enracinée et de diminuer notablement les pièges surinterprétatifs.

67 Si l'on convient qu'il n'y a pas de solution miracle à sortir d'un quelconque chapeau épistémologique, sans doute faudra-t-il se contenter de tels bricolages. Ce n'est déjà pas si mal. 


\section{NOTES}

1. II est certes plus facile de repérer les excès chez les autres : mais, bien évidemment, chacun d'entre nous a sans doute déjà été pris en flagrant délit de surinterprétation...

2. L. Boltanski avait, il y a longtemps, souligné le contraste entre le raisonnement juridique, qui se soucie de frontières et procède par dichotomies, et le raisonnement sociologique, qui pense (ou devrait penser) en termes de continuum et travaille sur des « ensembles flous » (L. Boltanski, Les cadres. La formation d'un groupe social, Paris, Éd. de Minuit, 1982, p. 256-264, 463 et sq.). L'absence de frontières nettes ne doit pas amener à nier la réalité de «foyers ". Notons au passage qu'un prochain numéro d'Enquête doit prendre comme objet les rapports entre raisonnement juridique et raisonnement sociologique.

3. La récente tentative d'U. Eco, tant dans Les limites de l'interprétation (Paris, Grasset, 1992) que dans Interprétation et surinterprétation (Paris, Presses universitaires de France, 1996) lorsqu'il argumente qu'on ne peut faire dire n'importe quoi à un texte, témoigne bien, malgré son apparente proximité avec notre propre tentative, de ce en quoi les problèmes sont d'ordre fort différent, puisqu'il est obligé, faute de référence possible à une démarche de recherche empirique, de recourir au concept quelque peu énigmatique de «l'intention de l'œuvre » (intentio operis) - sorte de droit du texte à être respecté en sa cohérence - comme garde-fou aux excès surinterprétatifs. En sciences sociales, la production d'un ensemble organisé et réfléchi de données empiriques est soumise à des contraintes, et crée à son tour des contraintes, qui ne sont pas du tout du même ordre que celles de l'écriture d'un roman ou d'un essai. Certes, on a pu vouloir réduire l'anthropologie à l'écriture anthropologique, et rabattre l'écriture anthropologique sur le lot commun des autres formes d'écriture. Mais c'est bien au prix exorbitant à mon avis - d'un oubli ou d'un déni des contraintes empiriques. Quant à la vision geertzienne de la culture comme texte, ce n'est guère qu'une formule, à faible productivité en termes de " programme de recherche».

4. On peut reprendre la définition de J.-C. Passeron : « forme particulière que prend la vérité dans l'exemplification empirique d'une proposition sociologique » (J.-C. Passeron, « De la pluralité théorique en sociologie: théorie de la connaissance sociologique et théories sociologiques ", Revue européenne des sciences sociales, XXXII, 98, 1994, p. 76).

5. Le titre de cet article est donc un raccourci quelque peu abusif : ce n'est pas aux «données » prises en elles-mêmes - que la surinterprétation fait violence, mais au substrat empirique en son ensemble, tel qu'il est défini ici.

6. Certaines de ces opérations ont été décrites par D. Sperber, dans Le savoir des anthropologues, Paris, Hermann, 1982 (cf. en particulier les interprétations d'interprétations - «indigènes » -, ainsi que les généralisations interprétatives). Nous ne rentrerons pas ici dans un débat beaucoup trop vaste sur l'interprétation en anthropologie.

7. Cf. J. Revel, ed., Jeux d'échelle : la micro-analyse à l'expérience, Paris, Gallimard/Seuil, 1995.

8. Sur certains usages du rapport entre « représentations » et "dispositifs », cf. J.-P. Olivier de Sardan, Anthropologie et développement. Essai en socio-anthropologie du changement social, Paris, Karthala, 1995, p. 150-152. On pourrait aussi évoquer la complémentarité entre «contraintes structurelles » et «contraintes contextuelles » chez Strauss (La trame de la négociation: sociologie qualitative et interactionnisme, Paris, L'Harmattan, 1992).

9. La réfutabilité, ici, ne se limite donc pas à l'infirmation/confirmation d'hypothèses théoriques (plus ou moins nomologiques), mais concerne tout démenti d'ordre empirique apporté à un énoncé anthropologique quel que soit son niveau d'interprétation (général ou spécifique, ou 
encore "proche" ou «loin» des données, pour plagier Geertz). Pour laisser à la notion de réfutabilité son sens poppérien (et expérimentaliste) rigoureux, nous parlerons plus prudemment de quasi-réfutabilité en ce qui concerne les sciences sociales.

10. Ainsi M. Augé, dans un livre récent, passe en revue différents modèles critiques qui structurent l'anthropologie contemporaine et cite le modèle des "évidences » (en fait dans le sens anglais du terme, proche de " preuves »), parlant à ce propos de "résistance des faits ", de «démenti des faits», d'«épreuve du réel», d'«opposer une évidence empirique à une interprétation théorique ", du "rapport entre un système d'interprétation et ses écarts » ( $M$. Augé, Pour une anthropologie des mondes contemporains, Paris, Flammarion, 1994, p. 65-67).

11. Un exemple éclairant, parmi bien d'autres, de ce en quoi bien des « savoirs traditionnels » ne sont pas des «systèmes » est donné par Last - «The Importance of Knowing about non Knowing ", Social Science and Medicine, 15 B, 1981, p. 387-392 - ; en l'occurrence il s'agit des savoirs thérapeutiques hawsa au Nord-Nigeria.

12. C'est pourquoi je préfère parler de «configurations» de représentations, et mettre en évidence leur caractère "modulaire ", par "paquets » ou par «familles », et la multiplicité des logiques correspondantes, bien loin à cet égard d'une logique unique, d'une logique des logiques ou « anthropologique » selon Augé (cf. M. Augé, Théorie des pouvoirs et idéologie, Paris, Hermann, 1975). Ceci, toutefois, ne signifie pas pour autant suivre Sperber sur son terrain cognitiviste, quand il « loge » des modules de représentations dans le cerveau : il y a là un tout autre problème (cf. D. Sperber, La contagion des idées, Paris, Odile Jacob, 1995).

13. Bien sûr, cette figure, comme toutes les autres, a déjà été identifiée depuis longtemps. Au sein de l'anthropologie contemporaine, outre le texte de Keesing dont la traduction figure dans ce numéro (p. 211-238), on peut évoquer P. Boyer qui s'attaque au "présupposé de cohésion» (P. Boyer, Tradition as Truth and Communication, Cambridge, Cambridge University Press, 1990, p. 3-4). 14. Dans l'anthropologie africaniste française, le plus célèbre exemple d'«obsession de la cohérence" est l'œuvre de Griaule et Dieterlen. La démonstration sereine mais implacable, accompagnée de la mise en évidence du processus qui y a conduit, se trouve dans W. van Beek, «Dogon Restudied: a Field Evaluation of the Work of Marcel Griaule », Current Anthropology, 32 (2), 1991, p. 139-158.

15. R. M. Keesing (cf. toujours dans ce même numéro p. 211-238) avait utilisé cette inversion des regards à propos du terme "luck» en anglais. B. Latour ("Comment redistribuer le grand partage ? ", Revue de Synthèse épistémologique, 110, 1983, p. 203-236) fait grand cas de ce procédé qu'il nomme "rectification de Bloor", en faisant allusion au texte de ce dernier qui, pour évoquer la nécessaire « symétrie » de la recherche, imagine un anthropologue zandé en Occident (D. Bloor, Sociologie de la logique, ou les limites de l'épistémologie [1976], Paris, Pandore, 1983).

16. Cas que je connais bien (et qui est, pour cette fois, américain) : P. Stoller - cf. « The Word and the Cosmos, "Zarma Ideology" Revisited », Bulletin de l'IFAN, 40 (4), 1978, p. 867 - prétend que « bonkaane » en songhay est un mot qui signifie à la fois «chef » et « chance ». Il s'empare de cette particularité sémiologique qui l'enchante pour développer une «théorie » du chef comme portebonheur collectif. Malheureusement l'oreille lui a fait défaut, car on a affaire à deux mots parfaitement distincts que nul locuteur songhay-zarma ne confondra jamais (si bonkaane «bonne tête " - signifie bien «chance ", c'est bon-koyne - «qui détient la tête » - qui signifie « chef »).

17. Cette question de la traduction en anthropologie a donné lieu à une vaste littérature; cf. entre autres J. Needham, Belief, Language and Experience, Oxford, Blackwell, 1972 ; et D. Sperber, Le savoir des anthropologues, op. cit. Bien évidemment, une bonne partie des débats relève de l'espace des interprétations empiriquement légitimes concurrentes. Mais il y aussi des traductions dont on peut «quasi-prouver » qu'elles font violence aux données émiques pour les besoins de leur argumentation. 
18. J'ai développé cette démonstration ailleurs - J.-P. Olivier de Sardan, « Possession, affliction et folie. Les ruses de la thérapisation ", L'Homme, 131, 1994, p. 7-27-, ainsi que proposé, toujours à propos des cultes de possession, l'analyse d'un autre type de surinterprétation, qui relève plutôt de la sous-figure 4 ci-dessous (l'imputation émique abusive) : cf. J.-P. Olivier de Sardan, «La surinterprétation politique : les cultes de possession hawka du Niger », in J.-F. Bayart, ed., Religion et modernité en Afrique noire. Dieu pour tous et chacun pour soi, Paris, Karthala, 1993.

19. Cf. le texte de Keesing publié dans ce numéro (p. 211-238), et G. Lakoff et M. Johnson, Les métaphores dans la vie quotidienne [1980], Paris, Éd. de Minuit, 1985.

20. L'ouvrage de J. C. Scott, Domination and the Arts of Resistance. Hidden Transcripts, New HavenLondres, Yale University Press, 1990, est pour une part une illustration de ce travers, dans le champ de l'anthropologie politique comparée.

21. Si D. Sperber (La contagion des idées, op. cit.) a le mérite de rappeler qu'une culture n'est rien d'autre qu'un ensemble de représentations partagées qui, en tant que représentations, ne sont qu'une forme particulière des représentations mentales individuelles, il fait l'impasse (concession au culturalisme ?) sur ce qui est le problème principal de l'anthropologie, à savoir les diverses formes de partage de ces représentations partagées; il suffit de rappeler que: (a) nombre de représentations culturelles ne sont qu'en partie seulement partagées; (b) elles coexistent le plus souvent avec d'autres représentations culturelles contradictoires, elles-mêmes en partie partagées...

22. E. Terray, «Une nouvelle anthropologie politique?», L'Homme, 110, 1989, p.5-29; cf. P. Clastres, La société contre l'État, Paris, Éd. de Minuit, 1974.

23. C. Lévi-Strauss, Anthropologie structurale, Paris, Plon, 1958, p. 311-317.

24. La généralisation abusive, à cet égard, n'est pas sans rappeler ce que Boudon appelle le « surclassement des énoncés » (un énoncé de possibilité devenant un énoncé de conjecture, et un énoncé de conjecture devenant un énoncé nomologique); cf. R. Boudon, La place du désordre, Paris, Presses universitaires de France, 1984.

25. Cf. J.-C. Passeron, op. cit., p. 203.

26. L'article ci-après de Bernard Lahire (p. 61-87) propose par exemple une typologie tri-partite de la surinterprétation autrement constituée, et manifestement complémentaire.

27. In M. Mauss, Sociologie et anthropologie, Paris, Presses universitaires de France, 1950.

28. Une nouvelle fois, je renvoie à l'article ci-après de Keesing (p. 211-238).

29. Cf. M. Kilani, « Que de hau! Le débat autour de l'Essai sur le don et la construction de l'objet en anthropologie ", in J.-C. Adam, M.-J. Borel, C. Calame et M. Kilani, Le discours anthropologique, Paris, Méridiens Klincksieck, 1990.

30. C. Lévi-Strauss, «Introduction à l'œuvre de Marcel Mauss ", in M. Mauss, op. cit.

31. Ainsi M. Godelier (L'énigme du don, Paris, Fayard, 1996), bien qu'il évoque, outre les arguments de Firth et de Sahlins, divers travaux océanistes contemporains, s'intéresse assez peu au rapport entre Mauss et son substrat empirique de référence. Il ne récuse d'ailleurs pas véritablement la soi-disante explication indigène du contre-don par l'âme contenue dans la chose donnée, mais la considère comme insuffisante, en particulier parce que reflétant une sorte de fausse conscience, un «fétichisme de la religion » analogue au « fétichisme de la marchandise » analysé par Marx. Son propos est clairement réinterprétatif : il s'attache à « compléter » Mauss sur un plan avant tout théorique et transculturel, afin de promouvoir sa propre solution à ce qu'il appelle l'énigme du don.

32. C'est en raison de tels effets que Collins a pu analyser le rapport entre deux grands types de «traditions» sociologiques, la tradition « héroïque loyaliste», et la tradition impersonnelle : R. Collins, «Les traditions sociologiques », Enquête, Anthropologie, histoire, sociologie, 2, Usages de la tradition, 1995, p. 11-38. 
33. «La théorie est générée par une confrontation constante avec les "cas négatifs", ceux qui ne confirment pas la formulation en cours. Le chercheur est ainsi engagé dans un processus de reformulation d'hypothèses et de redéfinition des phénomènes » (A. Strauss, op. cit., p. 286).

\section{RÉSUMÉS}

Considérons comme relevant de la surinterprétation tous les cas où apparaît une contradiction significative entre les références empiriques et les propositions interprétatives. En effet les sciences sociales, dans un espace épistémologique à la fois totalement interprétatif et empiriquement contraint, doivent légitimer leurs énoncés interprétatifs au nom d'un certain indice de véridicité, garanti par un double lien empirique : entre le «réel de référence " et les données produites à son sujet par les opérations de recherche, entre ces données et les énoncés proposés.

Bien qu'une délimitation précise de la frontière interprétation/surinterprétation soit impossible, on peut néanmoins repérer quelques foyers de surinterprétation, où apparaissent la projection excessive de préconception et le manque de vigilance méthodologique. Cinq figures, qui peuvent se combiner, sont ici examinées: la réduction à un facteur unique, l'obsession de la cohérence, l'inadéquation significative, la généralisation abusive, et le « coup du sens caché ».

Les pièges de la surinterprétation ne doivent pas empêcher la prise de risque interprétatif. En fait la surinterprétation est un moins-disant empirique, qui, en faisant violence aux données, refuse le défi qu'une prise en compte de la complexité de celles-ci adresse à l'imagination interprétative rigoureuse. Faute de recettes, la critique empiriquement fondée et la recherche des contreexemples invitent à produire des «modèles" plus exigeants en plausibilité empirique et en véridicité.

Let us consider all cases as those of overinterpretation where a significant contradiction appears between empirical references and interpretative propositions. Indeed, the social sciences, in an epistemological space that is entirely interpretative and empirically constrained at the same time, must legitimise their interpretative statements in the name of a certain mark of authenticity, guaranteed by a double empirical link : between the "real of reference " and the facts relating to it produced by research operations, between these facts and the statements proposed.

Although a clear demarcation of the border line interpretation/overinterpretation is impossible, one can nevertheless identify some centres of overinterpretation, where the excessive projection of preconceptions and the lack of methodological cautiousness appears. Five forms which can be combined are examined here : the reduction to a single factor, the obsession with coherence, the significant inadequacy, the abusive generalisation, and the « trick of hidden meaning ». 